\title{
Моделирование деятельности микропредприятий в Российских регионах
}

\section{Юлия Пиньковецкая}

Ульяновский государственный университет, г. Ульяновск, Россия

\section{Информация о статье}

Поступила в редакцию:

29.03.2019

Принята

к опубликованию:

27.05.2019

УДК 332.05

JEL L26, C31

\section{Ключевые слова:}

функция Кобба-Дугласа, микропредприятия, поток инвестиций, затраты на заработную плату, регионы России

\section{Keywords:}

Cobb-Douglas function, micro-enterprises, investment flow, payroll costs, Russian regions

\begin{abstract}
Аннотация
Целью исследования являлась оиенка двухфакторной производственной функиии, характеризующей взаимосвязь оборота микропредприятий от величины заработной плать работников и потока инвестиций в основной капитал. Рассмотрена производственная функиия, аналогичная функциии Кобба-Дугласа, без ограничений на сумму степеней при факторах. Исследование базировалось на статистических пространственных данных, использовалась информация по 82 регионам России за 2017 г. Производственная функиия представляет собой эффективный инструмент управления. Полученные новые знания имеют научное и практическое значение.
\end{abstract}

\section{Modeling of Microenterprises Activities \\ in the Russian Regions}

Iuliia Pinkovetskaia

\section{Abstract}

The goal of the research was to estimate the two-factor production function, which characterizes the relationship between the microenterprise turnover and the employees rate of wages and the flow of investments into the fixed assets.

The research examined a production function similar to that of Cobb-Douglas function, without the restrictions on the sum of degrees under factors. The research was based on statistical spatial data; using the information on 82 regions of Russia for 2017. The production function is an effective management tool. The new knowledge obtained is of scientific and practical importance. The methodological approach and tools proposed in the article for evaluating the production functions, describing the set of the microenterprises activities in the regions, can be applied in scientific research on the entrepreneurship issues, as well as in justifying the programs of this economy sector development at the federal and regional levels. The methodology and tools that were used in the research process can be applied in similar studies in the countries with a significant number of territorial (administrative) units. 
Further research is related to the evaluation of production functions for a set of microenterprises that are specialized in various types of economic activities, as well as those located in municipalities of specific regions.

\section{Введение}

В конце двадцатого века малое предпринимательство существенно увеличило свою долю в валовом внутреннем продукте и численности работников большинства развитых стран $[1,2,3]$. Малые предприятия, в том числе микропредприятия, выступают в качестве основных источников экономического роста, создания новых рынков, удовлетворение потребностей населения в рабочих местах $[4,5]$. Как показывает накопленный опыт $[6,7,8,9]$, именно предпринимательский сектор является основным драйвером регионального развития, особенно в слаборазвитых районах, и создает условия для структурной перестройки экономики. Поэтому малое предпринимательство стало в последние годы важнейшим элементом экономической политики как развитых, так и развивающихся стран.

В качестве объекта исследования нами были рассмотрены микропредприятия, расположенные в каждом из регионов России. Действующий закон [10] установил основной критерий отнесения хозяйствующих субъектов к микропредприятиям. Соответственно численность их работников не должна превышать 15 человек. В нашем исследовании изучались закономерности, характерные для совокупностей микропредприятий, расположенные в каждом из регионов.

На сегодняшний день в предпринимательском секторе России ведут деятельность многочисленные малые предприятия. В 2017 г. их число превышало 2755 тыс., в них работали 11986 тыс. человек. Среди общего количества малых предприятий преобладали микропредприятий, число которых достигало в 2017 г. 2498 тыс. В микропредприятиях была занята почти половина вех работников малых предприятий - 5314 тыс. человек. Доля малых предприятий (включая микропредприятия) составляет менее $20 \%$ валового внутреннего продукта и численности работников всех предприятий и организаций России. Таким образом, может быть сделан вывод о том, что малые предприятия пока не получили большого развития в России. Для сравнения можно отметить, что малые предприятия в странах Европейского Союза обеспечивают рабочими местами около $67 \%$ трудоспособного населения и производят 58\% валового внутреннего продукта.

Таким образом, в России в настоящее время имеется насущная потребность в ускоренном развитии микропредприятий. Развитие предпринимательского сектора национальной экономики требует понимания факторов, оказывающих влияние на деятельность микропредприятий и объемы их производства. Поэтому актуальной проблемой представляется определение резервов роста таких предприятий в каждом из регионов. Обоснование имеющихся резервов, а также ресурсов, необходимых для эффективного функционирования микропредприятий, может быть основано на таких математических моделях, как производственные функции. Отечественный и зарубежный 
опыт показал возможность широкого применения производственных функций в экономическом анализе и управлении. Производственные функции являются экономико-математическими моделями производственных процессов и количественно выражают устойчивую естественную зависимость между факторами [11], описывающими затраты капитала и труда, и показателем, характеризующим объем производства.

Исходя из этого, целью исследования, представленного в настоящей статье, являлась оценка факторов, оказывающих влияние на оборот микропредприятий, с использованием производственных функций.

\section{Обзор литературы}

Первые расчеты производственных функций были выполнены Ч. Коббом и П. Дугласом и представлены ими в 1928 г. в работе [12]. Теоретические основы, а также развитие теории и практики построения таких функций описаны в статье П. Дугласа [13]. В ней подчеркивается важность для развития теории производственных функций предложения Д. Дюрана [14] не накладывать ограничений на отдачу от масштаба производства. В России теоретические аспекты оценки и использования производственных функций нашли отражение во многих работах, среди которых наибольший интерес представляют $[15,16]$. В настоящее время наибольшее распространение получили степенные функции. В качестве объектов исследования в большинстве случаев выступают экономики регионов или России в целом. Исходными данными в абсолютном большинстве отечественных исследований являлись временные ряды, хотя в последнее время стали использоваться и пространственные данные. Вместе с тем оценка производственных функций по данным, характеризующим микропредприятия не получила развития в нашей стране. Можно отметить работу [17], в которой была подробно рассмотрена методика разработки производственных функций по данным совокупностей малых предприятий в регионах.

Исследования, основанные на производственных функциях по данным совокупностей малых предприятий, получили определенное развитие в зарубежных странах. В большинстве случаев факторами, которые определяют объем производства, являются капитальные затраты (стоимость всех машин, оборудования и зданий) и затраты труда. В качестве затрат труда в научных исследованиях обсуждаются разные показатели. Так в работах $[18,19]$ для описания трудозатрат используют количество постоянных сотрудников. А в книге [20] рассматривается такой показатель, как общее количество человеко-часов, отработанных в течение года. В большинстве работ наблюдения основаны на временных рядах. Так, в статье [21] доказано влияние количества рабочих и основного капитала на реальный ВВП по таким азиатским странам, как Бангладеш, Индии, Китая, Малайзии и Таиланда, на основе использования данных временных рядов за 1990-2014 гг. В работе [22] представлен анализ взаимосвязи этих же показателей на объем производства малых и средних предприятий в Пакистане. 


\section{Методический подход к оценке производственных функций}

В качестве объекта исследования нами были рассмотрены микропредприятия, расположенные в каждом из регионов России. Исследование показало, что в процессе оценки производственных функций, возникает ряд проблем, без преодоления которых не могут быть получены адекватные результаты. Остановимся на этих проблемах подробнее. Использование исходных данных за десять и более лет (временные ряды) осложняется тем, что необходимо учитывать имевшие место инфляционные процессы. Кроме того, необходимо исходить из предположения, что условия функционирования рассматриваемого объекта исследования за рассматриваемый интервал времени будут идентичны или, как минимум, претерпевать мало изменений, что на практике не всегда выполняется. Временные ряды зачастую ограничены по длине, тем более что в связи с кризисными явлениями в экономике динамика изменения показателей испытывает существенные флуктуации. Особенно большое влияние указанных тенденций при оценке функций, в которых имеются ограничения на сумму показателей степеней при факторах, то есть при постоянной отдаче от масштаба. При использовании в качестве фактора, описывающего затраты капитала, значений основных фондов, главной проблемой выступает достоверность информация относительно реально используемой в производственных процессах доли основных фондов рассматриваемой экономической системы. Предположение о полном использовании основных фондов не всегда соответствует фактической их загрузке. Аналогичная ситуация со вторым фактором. Численность работников, непосредственно занятых в производственных процессах, не всегда совпадает с фактическими трудозатратами, поскольку зачастую работники не заняты весь рабочий день (рабочую неделю). Это приводит к ошибочным показателям при оценке факторов труда. Наметившаяся в последние годы тенденция модификации производственных функций путем дополнения перечня факторов приводит к появлению мультиколлениарности, а также требует теоретического обоснования возможности их использования.

В российской статистике принят подход, согласно которому объем продукции, производимой микропредприятий, принято характеризовать суммарным оборотом, который складывается не только из товаров собственного производства, но и выручки от продажи приобретенных на стороне товаров. Учитывая задачи, стоящие в процессе оценки производственных функций, нами в процессе исследования рассматривалась только та часть оборота микропредприятий, которая связана со стоимостью товаров собственного производства, а также выполненных собственными силами работ и услуг.

Учитывая проведенный анализ, нами в качестве факторов производственной функции были рассмотрены инвестиции в основной капитал и заработная плата работников [17]. Корреляционный анализ показал, что эти факторы оказывают наибольшее влияние на оборот микропредприятий. При этом между ними нет взаимной связи (коллинеарности). Необходимо отметить, что поток инвестиций обеспечивает более приемлемые результаты по сравнению с таким фактором, как основные фонды. Такой вывод был сделан в работах В.А. Бессонова и С.В. Цухло [15], Е.Е. Гавриленкова [23], исходя из 
неполного использования основных фондов в микропредприятиях, о чем мы указывали ранее. Заработная плата работников, занятых в микропредприятиях представляет собой комплексный показатель, который учитывает не только затраты труда на производство продукции, но и особенности конкретного региона (уровень цен, занятость и другие социально-экономические аспекты). Кроме того, использование заработной платы работников в качестве фактора обеспечивает одинаковую размерность всех показателей производственной функции. Одинаковая размерность всех показателей производственной функции, как показано в статье [24] обеспечивает высокое качество построения соответствующих моделей.

В нашем исследовании были использованы пространственные данные, характеризующие рассматриваемые факторы и результирующие показатели по совокупности микропредприятий, расположенных в каждом из регионов России. Такой подход обусловлен следующим. Критерии отнесения предприятий к микропредприятиям в последние годы неоднократно менялись. Нынешние критерии используются с 2008 г. Бухгалтерский учет деятельности микропредприятий проводится один раз в год, соответственно ежегодно данные представляются в статистические органы. Поэтому моделирование показателей, характеризующих деятельность таких предприятий, с использованием временных рядов, возможно только на 10 лет (с 2008 по 2017 гг.). Соответственно, количество наблюдений равно девяти, что меньше минимально допустимого значения, которое в соответствии с критерием, который предложен в работе [25] должен для двухфакторной функции быть не менее 16. Поэтому производственная функция по совокупностям микропредприятий, построенные по временным данным, противоречат указанному критерию. Необходимо отметить, что пространственные данные позволяют уйти от проблем, которые характерны для временных рядов, о чем говорилось в литературном обзоре. Еще основоположник теории производственных функций П. Дуглас указывал, что интересно рассматривать много одновременно функционирующих объектов за один определенный промежуток времени. Достоинства использования пространственных данных при оценке производственных функций подробно описаны в работе [26].

Наше исследование включало следующие этапы: сбор и обработка первичных статистических данных по обороту, инвестициям в основной капитал и заработной плате совокупности микропредприятий, расположенных в каждом регионе; формирование информационных массивов сводных показателей; линеаризация полученных на первом этапе данных, характеризующих независимые факторы и результирующую переменную; разработка производственной функции с использованием метода наименьших квадратов.

Качество аппроксимации исходных данных функцией производилось на основе тестов Фишера-Снедекора и Стьюдента, а также соответствующих коэффициентов и уровней значимости. Проверка разработанной функции на наличие автокорреляции, гетероскедастичности и мультиколлинеарности, а также является ли распределение остатков по регрессии нормально распределенным с нулевым средним значением. 
В исследовании использовалась официальная статистика Федеральной службы государственной статистики [27] по деятельности малого предпринимательства в России за 2017 г. Исследование основано на информации по 82 регионам России. Таким образом, количество эмпирических наблюдений в процессе моделирования превышало предложенное в работе Харриса [28] оптимальное значение наблюдений равное 52. Некоторые характеристики показателей деятельности совокупностей микропредприятий, расположенных в регионах России, приведены в табл. 1.

Таблийа 1

Показатели деятельности микропредприятий за 2017 2., млрд руб.

\begin{tabular}{|l|c|c|c|c|c|}
\hline \multicolumn{1}{|c|}{ Показатели } & $\begin{array}{c}\text { Минимальное } \\
\text { значение }\end{array}$ & $\begin{array}{c}\text { Максимальное } \\
\text { значение }\end{array}$ & $\begin{array}{c}\text { Среднее } \\
\text { значение }\end{array}$ & $\begin{array}{c}\text { Медианное } \\
\text { значение }\end{array}$ & $\begin{array}{c}\text { Среднее квад- } \\
\text { ратическое } \\
\text { отклонение }\end{array}$ \\
\hline $\begin{array}{l}\text { Оборот совокупности мик- } \\
\text { ропредприятий в регионе }\end{array}$ & 4,365 & 5638,141 & 245,548 & 113,857 & 642,240 \\
\hline $\begin{array}{l}\text { Инвестиции в основной } \\
\text { капитал }\end{array}$ & 0,092 & 85,669 & 12,177 & 7,967 & 14,545 \\
\hline $\begin{array}{l}\text { Заработная плата работни- } \\
\text { ков микропредприятий }\end{array}$ & 1,132 & 1470,161 & 70,529 & 33,642 & 171,979 \\
\hline
\end{tabular}

Источник: составлено авторами

В процессе нашего исследования была разработана производственная функция, отражающая зависимость оборота микропредприятий от инвестиций в основной капитал и заработной платы работников по всем регионам России. Определение параметров производственной функции было выполнено с использованием методологии регрессионного анализа. Как уже отмечалось, оцениваемая функция описывает деятельность совокупностей всех микропредприятий, расположенных в каждом из регионов.

\section{Результаты экономико-математического моделирования}

Разработанная функция, описывающая зависимость оборот совокупности всех микропредприятий, расположенных в каждом из регионов, приведена далее:

$$
y_{1}\left(x_{1}, x_{2}\right)=4,476 \times x_{1}^{0,146} \times x_{2}^{0,840}
$$

где $y_{1}$ - оборот всех микропредприятий, расположенных в конкретном регионе России за год, миллиардов рублей;

$x_{1}$ - инвестиции в основной капитал всех микропредприятий этого региона за год, миллиардов рублей;

$x_{2}$ - заработная плата работников всех микропредприятий этого региона за год, миллиардов рублей.

Графическая интерпретация этой функции представлена на рис. 1. 


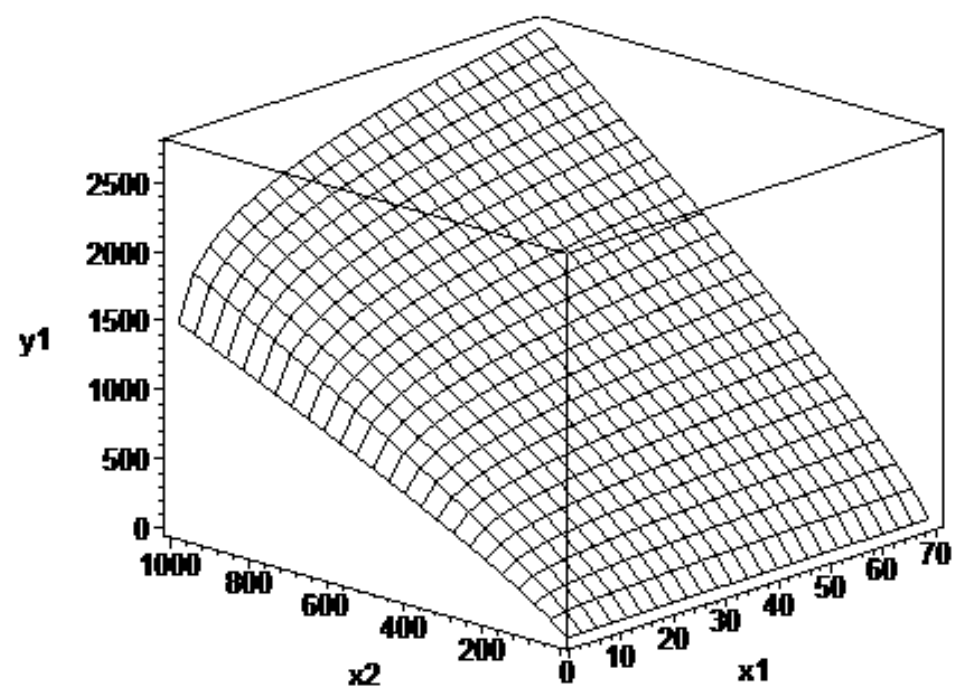

Рис. 1. Производственная функция (1)

Из рисунка видно, что рост заработной платы и инвестиций в основной капитал приводит к увеличению оборота совокупностей микропредприятий в регионах. Логический анализ производственной функции показал, что она адекватно описывает оборот микропредприятий на всем диапазоне изменения значений факторов.

Анализ качества полученной функции приведен в табл. 2. В ней представлены расчетные значения коэффициентов корреляции и детерминации, тестов Фишера-Снедекора и Стьюдента (столбец 2), также значимость по тесту Фишера-Снедекора и р-значения по тесту Стьюдента (столбец 3).

Таблийа 2

Расчетные значения по коэффициентам и тестам

\begin{tabular}{|l|c|c|}
\hline \multicolumn{1}{|c|}{ Показатели качества } & $\begin{array}{c}\text { Расчетные } \\
\text { значения }\end{array}$ & $\begin{array}{c}\text { Значимость } \\
\text { и р-значения }\end{array}$ \\
\hline \multicolumn{1}{|c|}{1} & 2 & 3 \\
\hline Коэффициент детерминации & 0,947 & - \\
\hline Коэффициент корреляции & 0,973 & - \\
\hline Стандартная ошибка & 0,292 & - \\
\hline Расчетное значение по тесту Фишера-Снедекора & 704,938 & меньше 0,001 \\
\hline Расчетное значение по тесту Стьюдента для $y$ - пересечения & 18,614 & меньше 0,001 \\
\hline Расчетное значение по тесту Стьюдента для первого фактора & 4,717 & меньше 0,001 \\
\hline Расчетное значение по тесту Стьюдента для второго фактора & 23,805 & меньше 0,001 \\
\hline
\end{tabular}

Сравнение расчетных значений, приведенных в таблице 2, со значениями тестов, представленных в литературе, показало, что производственная функция (1) обладает высоким качеством. Коэффициент корреляции близок к единице, что свидетельствует о близости к функциональной зависимости между объемом производства и двумя рассматриваемыми факторами. Известно, что функции обладают хорошим качеством и успешно описывают 
эмпирические данные, когда коэффициенты детерминации превышают 0,8. Для функции (1) этот коэффициент существенно больше критерия и составляет 0,982. Разница между единицей и коэффициентом детерминации характеризует долю дисперсии, которая обусловлена влиянием других факторов, не входящих в функцию. Это позволяет сделать вывод, что функция (1) объясняет 96,5\% вариации зависимых переменных. Соответственно, на другие факторы (которые здесь не учитываются) приходится не более 3,5\%. Расчетное значение статистики значительно выше табличного значения теста Фишера-Снедекора, который составляет 3,98 на уровне значимости, равном 0,05. Все расчетные значения теста Стьюдента больше табличного значения, которое при уровне значимости 0,05 составляет 1,99 . Таким образом, функция (1) хорошо аппроксимирует эмпирические данные. Интересно отметить, что высокое качество аппроксимации отмечалось и в проведенной ранее оценке производственной функции по обороту всех малых предприятий (включая микропредприятия), выполненной по данным за 2014 г. [29].

Расчетное значение уровня значимости теста Фишера-Снедекора меньше 0,001 (столбец 3 табл. 2). Это говорит о том, что действительно существует тесная корреляция между оборотом микропредприятий по регионам и такими факторами, как инвестиции в основной капитал этих предприятий и заработной платы их работников. Все р-значения меньше 0.001 , то есть с высокой достоверностью $(99,9 \%)$ коэффициент разработанной функции и показатели степеней в этой регрессионной модели статистически значимы.

Проверка функции (1) с использованием теста Дурбина-Уотсона показала отсутствие автокорреляции, а с использованием теста Бреуша-Пагана отсутствие гетероскедастичности. Уровень коллинеарности независимых переменных контролировался критерием дисперсионных факторов инфляции (VIF). Нормальность невязок доказана критериями нормальности ШапироВилка и Колмогорова-Смирнова. Делается вывод, что представленная модель (1) удовлетворяет эконометрическим допущениям и поэтому может быть использована для интерпретации.

Проверка функции (1) с использованием теста Дарбина-Уотсона показала отсутствие автокорреляции, а проверка по тесту Бреуша-Пагана - отсутствие гетероскедастичности. Мультиколлинеарности, то есть зависимости между факторами нет, что подтверждено тестом VIF.

В процессе аппроксимации исходных данных методом наименьших квадратов были получены остатки, показывающие отклонения расчетных значений от исходных данных. Проверка распределения этих остатков по производственной функции проводилась на основе оценки графика гистограммы, функции нормального распределения и тестов нормальности. Графическая интерпретация остатков регрессии, полученных в процессе моделирования, представлена на рис. 2. На нем показана гистограмма и соответствующая ей функция нормального распределения. 


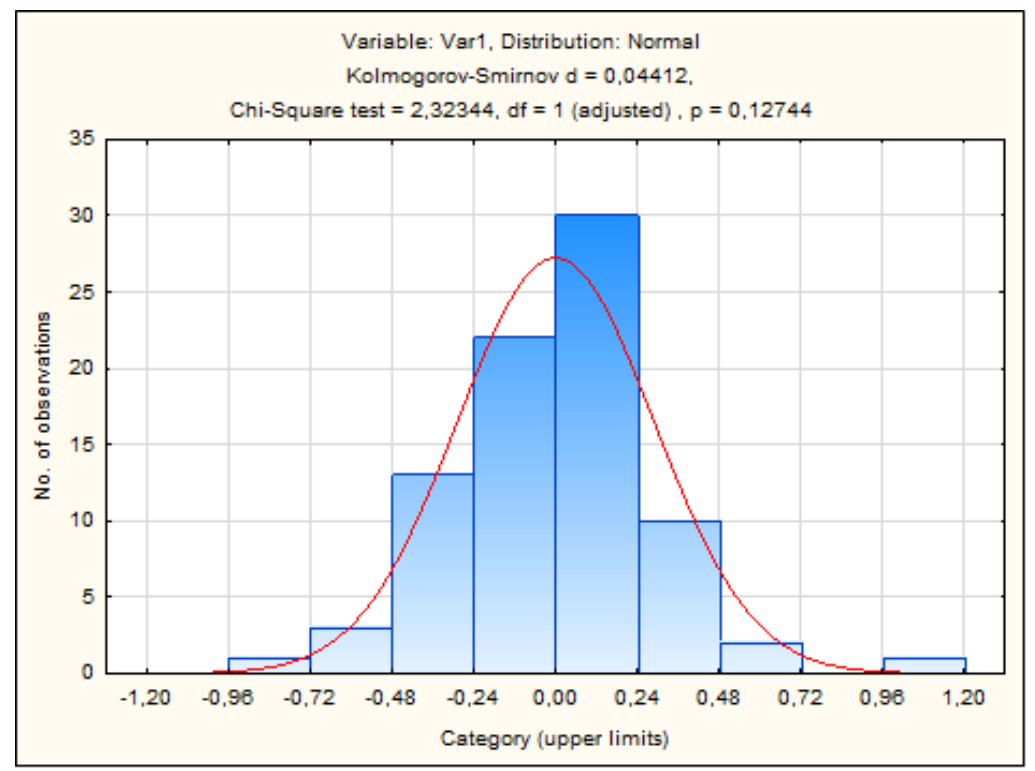

Puc. 2. Функция плотности распределения остатков регрессии

График гистограммы остатков характеризуется максимальными высотами построенных прямоугольников в середине гистограммы и минимальными высотами прямоугольников, расположенных на краях гистограммы справа и слева (так называемыми хвостами). Гистограмма симметрична относительно середины. Гистограмма демонстрирует, что остатки концентрируются около нуля. Этот же вывод следует из анализа функции распределения (2), которая имеет среднее значение равное нулю.

Функция распределения остатков $x_{3}$ приведена ниже.

$$
y_{2}\left(x_{3}\right)=\frac{19,68}{0,29 \cdot \sqrt{2 \pi}} \cdot e^{\frac{-\left(x_{3}-0\right)^{2}}{2 \times \cdot 0,29 \times 0,29}}
$$

Тесты Шапиро-вилка (расчетное значение 0,98 больше табличного 0,93), Пирсона (расчетное значение 2,32 меньше табличного 9,49) и Колмогорова-Смирнова (расчетное значение 0,04 меньше табличного 0,15) подтвердили нормальность распределения, описываемого этой функцией.

В целом можно сделать вывод, что разработанная функция (1) полностью удовлетворить эконометрическим требованиям и поэтому может быть использована описания сложившейся в регионах России зависимости оборота совокупностей микропредприятий от инвестиций в основной капитал и заработной платы работников этих предприятий.

\section{Обсуждение полученных результатов}

Разработанная производственная функция (1) доказывают наличие влияния рассматриваемых факторов на оборот предприятий, относящихся к микропредприятиям в регионах России. Значения степеней по обоим факторам в 
функции положительны, следовательно, можно констатировать, что стимулирование развития малого предпринимательства может быть обеспечено увеличением затрат на заработную плату и ростом инвестиций в основной капитал. Производственная функция для диапазона значений факторов (табл. 1) не достигают своего максимума. Это подтверждается тем, что значения предельной отдачи по обоим факторам положительны на рассматриваемых диапазонах изменения значений факторов. Следовательно, может быть сделан вывод о том, что экономика регионов России не достигла насыщения продукцией микропредприятий, и они имеют существенные резервы для дальнейшего развития. То есть во всех регионах имеются возможности наращивания количества предприятий и численности занятых в них работников.

Сумма значений показателей степеней в функции (1) меньше единицы, что указывает на убывающую отдачу от масштаба. С увеличением обоих факторов (инвестиций в основной капитал и заработной платы работников) рост объемов производства идет медленнее, чем рост факторов. Например, при росте обоих факторов в функции (1) на 10\% объем производства увеличивается на 9,86\%. Вместе с тем, для увеличения объемов производства микропредприятий в российских регионах целесообразно обеспечить одновременный рост обоих этих факторов. Следует отметить, что для регионов с избытком трудоспособного населения (на примере республик Северного Кавказа), основное направление развития предпринимательства связано с увеличением занятости и созданием семейного бизнеса. В регионах, где не хватает потенциальных работников (Сибирь и Дальний Восток), основное направление увеличения объемов производства связано с увеличением инвестиций в основной капитал. Перекрестные производные производственной функции для каждого из двух факторов положительны для всех значений диапазона изменяющихся факторов, поэтому увеличение одного из факторов улучшает условия использования другого фактора. Таким образом, рост заработной платы работников повышает отдачу от инвестиций в основной капитал. И, наоборот, при увеличении инвестиций в основной капитал возрастает уровень использования заработной платы. Вторые производные всех изоквант являются положительными. Отметим, что указанные выводы были характерны для производственной функции по микропредприятиям по данным за 2015 г. [11]. Уровень выпуклости снижается с ростом оборота, что свидетельствует об увеличении эластичности замещающих факторов: с ростом объемов производства в предпринимательских структурах возрастает возможность замещения одного фактора другим. Фактор заработной платы работников в производственной функции влияет на оборот в большей степени, чем фактор инвестиций в основной капитал.

Использование производственной функции возможно при решении такой насущной проблемы, как составление рейтинга регионов исходя из эффективности использования таких ресурсов, как инвестиции в основной капитал и заработная плата работников микропредприятий. При этом может использоваться сравнительный анализ достигнутого в регионе фактического оборота микропредприятий и предсказанного на основании производственной функции значения оборота в этом же регионе. На наш взгляд относи- 
тельно большое положительное значение этой величины (то есть превышение фактического оборота над расчетным) свидетельствует о хорошем предпринимательском климате в соответствующем регионе. И соответственно большое отрицательное значение этой величины позволяет сделать вывод о наличии проблем с предпринимательским климатом в соответствующем регионе.

Проведенный сравнительный анализ эмпирических данных, использованных при разработке производственной функции (1) и предсказанных значений по этой же функции, показал высокий уровень предпринимательского климата в Ивановской и Калининградской областях, городе Москве, а также Краснодарском крае. Низкий уровень предпринимательского климата по критерию эффективности использования рассматриваемых факторов отмечен в таких регионах, как Оренбургской, Кемеровской и Амурской областях, республике Коми, а также Хабаровском крае.

\section{Заключение}

Проведенные исследования имеют определенную научную и практическую значимость.

Научная значимость проведенного исследования заключается в следующем:

- рассмотрены методические аспекты оценки производственных функций. Проанализированы проблемы, вытекающие при использовании основных фондов в качестве фактора капитала и количества работников, занятых в производственных процессах в качестве фактора труда, а также данных, сформированных в виде временных рядов. Показаны преимущества выбора в качестве факторов при оценке производственной функции совокупностей микропредприятий в регионах потока инвестиций и заработной платы работников и пространственных данных за один год;

- в процессе исследований была разработана двухфакторная производственная функция, аналогичная функции Кобба-Дугласа. Эта функция описывает зависимость оборота микропредприятий от рассматриваемых факторов в регионах. С использованием ряда тестов было подтверждено высокое качество разработанной производственной функции и хорошую аппроксимацию исходных данных;

- производственная функция доказала, что имеются значительные резервы для дальнейшего развития предпринимательского сектора экономики, а именно, во всех российских регионах не достигнуто насыщение товарами и услугами микропредприятий. Рост каждого из факторов функции стимулирует эффективность использования соответственно другого фактора. С увеличением оборота в совокупности микропредприятиях улучшаются возможности замещения одного фактора другим. Заработная плата в полученной функции влияет на объем производства в существенно большей степени, чем поток инвестиций в основной капитал. По совокупностям микропредприятий в регионах наблюдается убывающая отдача от масштаба;

- с использованием производственной функции были определены регионы России с высоким и низким уровнем эффективности использования имеющихся ресурсов (предпринимательского климата). 
Практическая значимость проведенных исследований может быть реализована в деятельности органов власти, предпринимательском секторе национальной экономики, а также в образовательной деятельности при подготовке бакалавров и магистров, а также подготовке специалистов по проблемам малого и среднего предпринимательства.

Предложенный в статье методический подход и инструменты оценки производственных функций, описывающих деятельность совокупностей микропредприятий в регионах, могут быть использованы в научных исследованиях по проблемам предпринимательства, а также обоснования программ развития этого сектора экономики на федеральном и региональном уровнях. Методология и инструментарий, которые были использованы в исследовательском процессе, могут быть применены в аналогичных исследованиях в странах со значительным количеством территориальных (административных) единиц.

Проведенное исследование обеспечивает правительство, органы регионального управления и другие административные структуры информацией о возможных путях увеличения объемов производства микропредприятий. Разработанная производственная функция представляет собой эффективный инструмент управления, который позволяет проводить оценку уровня использования финансовых и трудовых ресурсов микропредприятий в России и конкретных регионах. Результаты работы могут быть использованы в текущей деятельности государственных, муниципальных и общественных организаций, связанной с регулированием и поддержкой малого и среднего бизнеса, в том числе корректировать свои действия на основе научных данных.

Практическая значимость исследования заключается в возможности использования полученных результатов для обоснования ресурсов и мониторинга предпринимательского климата. Результаты работы могут использоваться в научных исследованиях, диагностике состояния предпринимательского климата, обосновании наиболее эффективных объемов трудовых и инвестиционных ресурсов, установления ключевых индикаторов при разработке планов и программ развития предпринимательства. То есть для оценки уровня эффективности каждого из обсуждаемых факторов, а также выявленного дисбаланса в значениях факторов по каждому из регионов. Результаты исследования могут быть использованы при реализации Федеральной стратегии развития малого предпринимательства на период до 2030 г. [30].

Дальнейшие исследования связаны с оценкой производственных функций по совокупностям микропредприятий, которые специализированы на различных видах экономической деятельности, а также расположенных в муниципальных образованиях конкретных регионов.

\section{Список источников / References}

1. Wennekers S., Uhlaner L., Thurik R. Entrepreneurship and its conditions: a macro perspective // International Journal of Entrepreneurship Education. 2002. No. 1(1). P. 25-64.

2. Brock W.A., Evans D. Small business economics // Small Business Economics. 1989. No. 1. P. 7-20. 
3. Action Plan to Promote Entrepreneurship and Competitiveness, Directorate-General for Enterprise. The European Commission - DG Enterprise and Industry. Luxembourg: Eur-Op, 1999. 19 p.

4. Van Praag C., Versloot P. What is the value of entrepreneurship? A Review of Recent Research, Small Business Economics, 2007. No. 29(4). P. 351-382.

5. Feldman M., Lanahan L., Miller J. Inadvertent infrastructure and regional entrepreneurship policy. In M. Fritsch (Ed.), Handbook of research on entrepreneurship and regional development. Cheltenham: Edward Elgar. 2011. P. 216-251.

6. Мосина Е.А. Региональное малое предпринимательство: необходимые условия и перспективы развития // Социальная политика и социология. 2016. Т. 15. № 1. C. $17-23$.

7. Сафиуллин Р.Г., Гришина Т.П., Маликова Э.Р. Территориальная динамика конкурентоспособности малого предпринимательства в РФ // Успехи современного естествознания. 2016. № 11-2. С. 390-395.

8. Чепуренко А.Ю. Совмещая универсальные концепции с национальной спецификой: поддержка малого и среднего предпринимательства // Вопросы государственного и муниципального управления. 2017. № 1. С. 7-30.

9. Decker R., Haltiwanger J., Jarmin R., Miranda J. The Role of Entrepreneurship in US Job Creation and Economic Dynamism // Journal of Economic Perspectives. 2014. Vol. 28. № 3. P. 3-24.

10. О развитии малого и среднего предпринимательства в Российской Федерации [Электронный ресурс]: Федеральный закон от 24.07.2007 № 209-Ф3. URL: http://www.consultant.ru/document/cons_doc_LAW_52144/ (дата обращения: 10.12.2017).

11. Пиньковецкая Ю.С. Моделирование деятельности совокупностей малых и средних предприятий с использованием производственных функций // Человек. Общество. Инклюзия. 2017. № 3(20). С. 92-100.

12. Cobb C.W., Douglas P.H. A Theory of Production // The American Economic Review. 1928. Vol. 18. P. 139-165.

13. Douglas P. Are There Laws of Production? // The American Economic Review. 1984. Vol. 38. No. 1. P. 1-41.

14. Durand D. Some Thoughts on Marginal Productivity with Special Reference to Professor Douglas' Analysis // Journal of Political Economy. 1937. Vol. 45. P. 740-758.

15. Бессонов В.А., Цухло С.В. Проблемы построения производственных функций в российской переходной экономике // Анализ динамики российской переходной экономики. М.: Институт экономики переходного периода. 2002. С. 5-89.

16. Клейнер Г.Б. Производственные функции: Теория, методы, применение. М.: Финансы и статистика. 1986. 239 с.

17. Пиньковецкая Ю.С. Некоторые результаты моделирования объемов производства предпринимательских структур // ЭТАП: экономическая теория, анализ, практика. 2014. № 2. C. 107-126.

18. Bohórquez V., Esteves J. Analyzing SMEs size as a moderator of ERP impact in SMEs productivity // Communications of the IIMA. 2008. no. 8(3).

19. Husain S., Islam M.S. A Test for the Cobb Douglas Production Function in Manufacturing Sector: The Case of Bangladesh", International Journal of Business and Economics Research. 2016. no. 5(5). P. 149-154.

20. Sage A.P., Rouse W.B. Economic systems analysis and assessment cost, value, and competition in information and knowledge intensive systems, organizations, and enterprises. John Wiley \& Sons, New York, USA. 2011. 
21. Khatun T., Afroze S. Relationship between real GDP and Labour and Capital by applying the Cobb-Douglas production function: a comparative analysis among selected Asian Countries // Journal of Business Studies. 2016. vol. XXXVII. no. 1. P. 113-129.

22. Batool S., Zulfiqar S. Analyzing the Input Output Relationship of Small and Medium Enterprises in Pakistan: An Econometric Approach // International Journal of Business and Economic Development. 2013. no. 1(1). P. 66-73.

23. Гавриленков Е.Е. Экономический рост и долгосрочная стратегия развития России // Российская экономика: опыт трансформации 1990-х годов и перспективы развития. М.: ГУ-ВШЭ, 2000. С. 55-78.

24. Felipe J., McCombie J. Problems with Regional Production Functions and Estimates of Agglomeration Economies: A Caveat Emptor for Regional Scientists // Cambridge Centre for Economic and Public Policy. 2012. Working Paper no. 725.

25. Ходасевич Г.Б. Обработка экспериментальных данных на ЭВМ. Часть 2: Обработка одномерных массивов [Электронный pecypc]. URL: http://dvo.sut.ru/libr/opds/i130hod2/index.htm (дата обращения: 12.10.2018).

26. Charoenrat T., Harvie C. Technical Efficiency of Thai Manufacturing SMEs: A Stochastic Frontier Analysis", Australasian Accounting. Business and Finance Journal. 2013. no. 7(1). P. 97-122.

27. Федеральная служба государственной статистики. Малое и среднее предпринимательство России.

URL: http://www.gks.ru/wps/wcm/connect/rosstat_main/rosstat/ru/statistics/publications/catal og/doc_1139841601359 (дата обращения: 15.01.2018).

28. Harris R.J. A primer of multivariate statistics. New York: Academic Press. 1985. 632 p.

29. Пиньковецкая Ю.С. Модели объемов производства совокупностей малых и средних предприятий в субъектах Российской Федерации // Мир новой экономики. 2016. № 2. С. 113-118.

30. Стратегия развития малого и среднего предпринимательства в Российской Федерации на период до 2030 года [Электронный ресурс]: Распоряжение Правитель$\begin{array}{lllll}\text { ства от № } & \text { о } 06.2016 & \text { URL: }\end{array}$ http://www.consultant.ru/document/cons_doc_LAW_199462/f3fa9da4fab9fba49fc9e0d 938761 ccffdd288bd/ (дата обращения: 21.12.2017).

\section{Сведения об авторе / About author}

Пиньковецкая Юлия Семеновна, кандидат экономических наук, доцент, доцент кафедры экономического анализа и государственного управления, Ульяновский государственный университет. 432017 Россия, г. Ульяновск, ул. Льва Толстого, 42. E-mail: judy54@yandex.ru Iuliia S. Pinkovetskaia, Candidate of Economical Sciences, Associate Professor, Associate Professor of the Department of Economic Analysis and Public Administration, Ulyanovsk State University. 42 L. Tolstogo str., Ulyanovsk, Russia 432017. E-mail: judy54@yandex.ru 